\title{
Scheduling Advantages of Network Coded Storage in Point-to-Multipoint Networks
}

\author{
Ulric J. Ferner ${ }^{\dagger}$, Parastoo Sadeghi ${ }^{\ddagger}$, Neda Aboutorab ${ }^{\ddagger}$, and Muriel Médard ${ }^{\dagger}$ \\ ${ }^{\dagger}$ Research Laboratory for Electronics, Massachusetts Institute of Technology, Cambridge, MA 02139, USA \\ ${ }^{\ddagger}$ Research School of Engineering, Australian National University, Canberra ACT 0200, Australia
}

\begin{abstract}
We consider scheduling strategies for point-tomultipoint (PMP) storage area networks (SANs) that use network coded storage (NCS). We present a simple SAN system model, two server scheduling algorithms for PMP networks, and analytical expressions for internal and external blocking probability. We point to select scheduling advantages in NCS systems under normal operating conditions, where content requests can be temporarily denied owing to finite system capacity from drive I/O access or storage redundancy limitations. NCS can lead to improvements in throughput and blocking probability due to increased immediate scheduling options, and complements other well documented NCS advantages such as regeneration, and can be used as a guide for future storage system design.
\end{abstract}

\section{INTRODUCTION}

The prolific growth of online content and streaming video makes serving content requests to multiple users simultaneously an important technique for modern storage area networks (SANs). Two fundamental measures of service quality are system external blocking probability, i.e., the probability that a requesting user is denied immediate access to content, as well as system throughput. Under normal operating conditions and given perfect scheduling, network coded storage (NCS) has been identified as a promising technique to reduce blocking probability. For instance, [1] used queuing theory to show that network coding can reduce system blocking probability. In this paper we build upon this idea and develop simple and intuitive server scheduling algorithms for such NCS systems. We then explore their impact on both throughput as well as blocking probability. The main contributions of this paper are:

- We introduce a simple storage model for point-tomultipoint (PMP) storage networks that allows direct evaluation of blocking probability and system throughput;

- Using this model, we propose two intuitive scheduling algorithms - one for uncoded storage (UCS) and one for NCS - that can achieve maximal throughput;

- We quantify the blocking probability and throughput savings of NCS over UCS scheduling, showing that a small improvement in throughput translates to a comparatively large improvement in blocking probability.

This paper builds upon and complements existing work in this area. The use of NCS as regenerating codes is a well studied repair technique to enhance SAN reliability [2]

This material is based upon work supported by the Martin Family Society of Fellows for Sustainability at MIT, by BAE Systems National Security Solutions Inc., under award 739532-SLIN 0004, and the Australian Research Councils Discovery Projects funding scheme (project no. DP120100160). in both centralized and distributed systems. This particularly holds in less common operating conditions, such as permanent drive failures. In modern systems traffic-induced temporary unavailability significantly dominates disk failures [3], and so like in [1], this paper focusses on normal operating conditions and seeks to avoid highly transient and temporary bottlenecks in data liveness. General scheduling for coded storage in pointto-point networks, when users are served sequentially instead of simultaneously, are considered in [4], [5].

Server scheduling is also well studied in matched networks such as cross-bar switches. Throughput-optimal schedules are considered for $N \times M$ point-to-point cross-bar switches using graph theory and techniques such as the Birkhoff-von Neumann theorem [6]. Switches with multicast and broadcast capabilities with a queueing analysis flavor are considered in [7]. References [8], [9] attempt to map the multicast problem in cross-bar switches to simpler problems such as blockpacking games and round-robin based multicast. By characterizing flow conflict graphs and their corresponding stable set polytopes in multicast cross-bar switches, [18] proposed online and offline network coding schedules for enhancing throughput. For general PMP storage networks, developing appropriate storage models, corresponding conflict graphs, and throughput optimal scheduling is an interesting and largely unaddressed area of research. This paper takes a first step towards this by considering a particular kind of PMP network, namely broadcast, and by developing intuitive coded and uncoded leader-based scheduling, which do not explicitly require conflict graph construction. Chunk scheduling problems in uncoded peer-to-peer networks, as opposed to PMPs, are considered in [10], and for star-based broadcast networks in [11]. Note also that unlike classical asynchronous broadcast problems [12], [13], our goal is not to reduce content delivery delay or to optimize caching. Instead, by taking into account intermittent drive availability, we aim to determine the impact of scheduling drive reads and the impact of content storage format on blocking probability and throughout. We expect that by using appropriate caching, performance can be further improved. However, this is beyond the scope of this work.

The remainder of this paper is organized as follows. Section II details our system model. Section III presents service schemes and Section IV describes numerical results. Section $\mathrm{V}$ concludes the paper. 


\section{International Symposium on Network Coding (NetCod)}

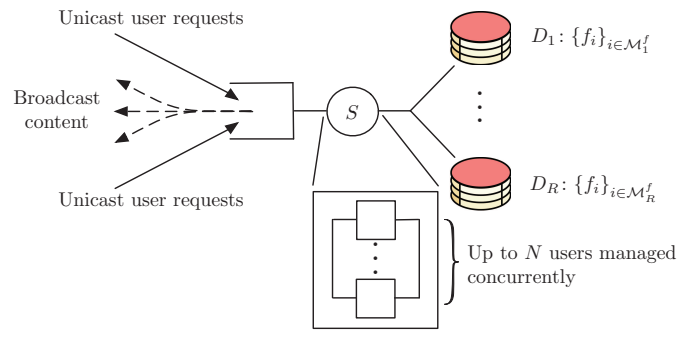

Fig. 1. System model.

\section{System MOdEL}

Fig. 1 depicts our tree-structured connectivity model made of single server $S$, connected to $R$ drives, that receives user requests for content.

\section{A. Drives}

The SAN in Fig. 1 stores a single chunked file $\mathcal{F}=$ $\left\{f_{1}, f_{2}, \cdots, f_{T}\right\}$, where $T$ is the number of chunks in $\mathcal{F}$, and $\mathcal{F}$ is stored across a set of drives $\mathcal{D}=\left\{D_{1}, \cdots, D_{R}\right\}$. ${ }^{1}$ If drive $D_{i}$ receives a read request for chunk $f_{j}$, and if $D_{i}$ stores $f_{j}$ and $D_{i}$ is available, then it takes one timeslot to read out that content and broadcast to all users. We model the overall effect of drives having finite I/O access bandwidth with parameter $P_{b}^{D}$, where $P_{b}^{D}$ is the probability that any drive is blocked in timeslot $t$. For simplicity, we assume drives are blocked independently of one another and across timeslots. ${ }^{2}$

In UCS, let $\mathcal{M}_{i}^{d} \subseteq \mathcal{D}$ be the collection of drives that hold uncoded file chunk $f_{i}$ and conversely, let $\mathcal{M}_{i}^{f} \subset \mathcal{F}$ be the collection of file chunks held by drive $D_{i}$. The only requirement of chunks to drives is that $R$ drives collectively hold at least one copy of the whole file, i.e., $\mathcal{F}=\cup_{i=1}^{R} \mathcal{M}_{i}^{f}$.

In NCS, the $r$ th coded file chunk is represented as [1]

$$
c_{r}=\sum_{j=1}^{T} \alpha_{j, r} f_{j}
$$

where $\alpha_{j, r}$ is the encoding coefficient of file chunk $f_{j}$ and the corresponding encoding vector is

$$
\mathbf{k}_{r}=\sum_{j=1}^{T} \alpha_{j, r} \mathbf{e}_{j}
$$

In (2), $\mathbf{e}_{j}=\left[e_{j, 1}, \cdots, e_{j, T}\right]$ is the unit encoding row vector of length $T$ with elements $e_{j, r}=\delta_{j, r}$. Function $\delta_{j, r}$ is the Kronecker delta function with $\delta_{j, r}=1$ iff $j=r$. We assume that a total of $H$ linearly coded chunks $c_{1}$ to $c_{H}$ are stored onto drives via some MDS code, such that any $T$ coded chunks are linearly independent so that the original file chunks can be recovered from them using Gaussian elimination. If encoding coefficients $\alpha_{j, r}$ are randomly selected from a finite field $\mathbb{F}_{q}$

\footnotetext{
${ }^{1} \mathcal{F}$ may represent one or more logical physical files.

${ }^{2}$ This blocking model can be applicable where other servers have access to the same drives and therefore, there is some probabilistic traffic-induced blocking observed by $S$. More realistic models for traffic-induced drive blocking as well as more general PMP traffic patterns are beyond the scope of this paper and subject of our current research.
}

with sufficiently large size $q$, this requirement is satisfied with high probability [14].

B. Server

We assume server $S$ has a bounded buffer to manage concurrent user requests. Let $N$ be the maximum number of users that can be managed and serviced concurrently and suppose $S$ operates in slotted time. In particular, in any timeslot, $S$ can serve at most $N$ active requests for content. A user request for a content is cleared from the buffer when all its requested file chunks have been transmitted by $S$.

Any additional request beyond $N$ for the same content will be externally blocked. ${ }^{3}$ We will discuss the relation between external and internal blocking in Section II-D. This is a similar model to existing drive blocking models [1] and existing practical server experimentation test [15]. When a user request arrives and is not externally blocked, one slot of the server buffer is allocated to manage and service this user request. We make the following additional assumptions about how $S$ retrieves content from $\mathcal{D}$ :

- Let the vector $\boldsymbol{b}(t)$ of size $R$ be the drive availability vector, where $b_{i}(t)=0$ means drive $D_{i}$ is free for reads and $b_{i}(t)=1$ means it is busy in timeslot $t$. We assume that $\boldsymbol{b}(t)$ can be obtained by the server at the beginning of timeslot $t$ with negligible time overhead.

- In timeslot $t$, based on $\mathbf{b}(t), S$ can choose to send a read request to access a single drive and read a single chunk.

- At the end of timeslot $t, S$ broadcasts the received chunk $x(t)$ to users active in the buffer.

We assume perfect communication so when $S$ broadcasts content all active users receive that content without error.

\section{Users}

We model users with the following key parameters:

- User requests arrive at $S$ following a Poisson process with rate $\lambda$.

- All user requests are for the entire file $\mathcal{F}$, so in the longterm there is uniform traffic demand across file chunks.

- Users currently being managed and serviced by $S$ are referred to as active users, which we denote by $\mathcal{U}_{A}$, which is a subset of all serviceable users $\mathcal{U}=\left\{u_{m}\right\}$.

Each user $u_{m}$ stores the received encoding vectors up to timeslot $t$ in a buffer (matrix) denoted by $\mathbf{K}_{m}(t)$. This is called the knowledge space of user $u_{m}$ at timeslot $t$. The rank of knowledge space of user $u_{m}$ at timeslot $t$ is denoted by $r_{m}(t)=\operatorname{rank}\left(\mathbf{K}_{m}(t)\right)$.

A user is said to receive a new degree of freedom (d.o.f.) if the rank of its knowledge space increases by one after reception of a chunk from $S$, that is, if $r_{m}(t+1)=r_{m}(t)+1$. A file chunk $f_{j}$ is said to decoded by user $u_{m}$ if the user can obtain the corresponding unit encoding vector $\mathbf{e}_{j}$ (possibly after Gaussian elimination) from its knowledge space $\mathbf{K}_{m}(t)$.

An active user $u_{m}$ at timeslot $t$ is a user whose d.o.f. satisfies $r_{m}(t)<T$. User $u_{m}$ is said to depart the queue

${ }^{3} N$ is an arbitrary, possibly time varying, quantity and hence this model does not limit our analysis. 


\section{International Symposium on Network Coding (NetCod)}

at time $t$ when the rank of its knowledge space becomes $T$. Throughout the rest of the paper, a user always refers to an active user who has not yet departed from the server's buffer.

References [1], [2] have assumed perfect scheduling by the server, which is not assumed in our model. Somewhat related to this issue is the assumption that the coefficients of a coded chunk are cycled or refreshed to ensure innovative chunks for every drive read. Finally, to be able to apply queuing theoretical arguments in [1], requests for different file chunks of the same content arrive randomly and independently of other chunks at the server. In that paper, the notion of users is abstracted away, which we do not do here.

\section{Performance Metrics}

Let $\mathcal{U}_{n}(t) \subset \mathcal{U}_{A}$ be the subset of targeted users who receive an innovative d.o.f. from the broadcast of chunk $x(t)$ at timeslot $t$. We define three throughput metrics in order of strongest to weakest, which are equivalent to those used in cross-bar switch scheduling [16].

Definition 1. (Throughput optimal) A scheduling service is throughput optimal if every service can guarantee $\mathcal{U}_{n}(t)=\mathcal{U}_{A}$. That is, $r_{m}(t+1)=r_{m}(t)+1, \forall u_{m} \in \mathcal{U}_{A}, \forall t$.

Since system constraints may mean that throughput optimality is not feasible, we consider maximum and maximal throughput, which are in general the best any scheduling scheme can do up to or at any timeslot based on constraints such as drive availability.

Definition 2. (Maximum throughput) A service scheme achieves maximum throughput if the total number of targeted users up to time $t$, denoted by $\sum_{i=1}^{t}\left|\mathcal{U}_{n}(t)\right|$ is maximized, across all service schemes for a given data storage allocation.

Definition 3. (Maximal throughput) A scheduling service achieves maximal throughput if at each timeslot $t$, the number of targeted users $\left|\mathcal{U}_{n}(t)\right|$ is maximized, across all service schemes for a given data storage allocation.

Note that any service scheme that achieves maximal throughput is necessarily a greedy algorithm. In a given timeslot, active users that are not targeted by a scheduling scheme are said to be internally blocked. These users are not externally blocked as they are already in the server's buffer, but are held up for service. The better the throughput of a scheduling scheme, the lower its internal blocking probability will be. Intuitively, a lower internal blocking probability should lead to lower external blocking probability as active users are flushed out of the system faster.

\section{Data Scheduling Schemes}

We introduce the concept of a service leader and considers two system types. First, to develop intuition for our problem and to verify expectations, we consider systems in which drives never block, i.e., drives with infinite I/O access bandwidth. Second, we consider systems with traffic-induced drive blocking, i.e., drives with finite I/O access bandwidth. In both systems, we propose service schemes for UCS and NCS. Schemes presented in this section can be formulated as integer linear programs over content demand graphs, similar to those for cross-bar switches [16], [17], but are omitted here.

\section{A. Infinite I/O access bandwidth systems}

To verify expectations, consider a system in which drives have infinite I/O access bandwidth, so $P_{b}^{D}=0$.

1) Uncoded Scheme: Consider UCS and the scheme outlined in Algorithm 1. We introduce the following terminology for our leader-based scheme, which will also be used in the finite I/O access bandwidth case. Let $\mathbf{a}_{m}(t)$ be a binary valued decoded chunk vector of length $T$ for user $u_{m}$ with elements $a_{m, j}(t)$. If $a_{m, j}(t)=0$ then user $u_{m}$ has decoded file chunk $f_{j}$ and if $a_{m, j}(t)=1$ then file chunk $f_{j}$ is yet to be decoded. Upon arrival of user $u_{m}$ 's file request, $a_{m, j}(t)=1$ for all $1 \leq j \leq T$ and upon departure $a_{m, j}\left(t^{\prime}\right)=0$ for all $1 \leq j \leq T$.

- The leader user $u_{\ell}$ at timeslot $t$ is the user with maximum knowledge space rank. That is,

$$
\ell=\operatorname{argmax}_{m: u_{m} \in \mathcal{U}_{A}} r_{m}(t) .
$$

- The earliest undecoded chunk or simply min chunk of user $u_{m}$ is the chunk for which $a_{m, j}(t)=1$ and all $a_{m, k}(t)=0$ for $k<j$.

- The earliest undecoded chunk of the leader or simply min-max chunk $f_{j^{*}}$ is the chunk for which $a_{\ell, j^{*}}(t)=1$ and $a_{\ell, k}(t)=0$ for $k<j^{*}$ for the leader user $u_{\ell}$.

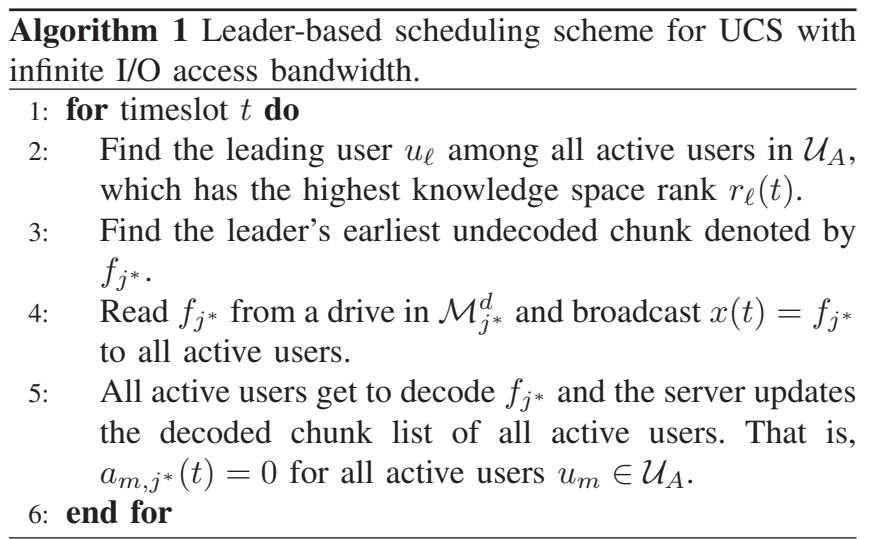

2) Uncoded Scheme Analysis: Intuitively a system with infinite $\mathrm{I} / \mathrm{O}$ access bandwidth and perfect communications will allow for throughput optimal scheduling by $S$, since the scheme is without errors. We now formalize that the leaderbased scheme in Algorithm 1 is throughput optimal according to Definition 1.

Lemma 1. The scheduling scheme of Algorithm 1 is throughput optimal.

Most proofs in this paper are omitted due to space limitation and can be found in [19].

Lemma 2. If a scheduling scheme is throughput optimal, then it also minimizes the blocking probability across all feasible scheduling schemes.

Applying Lemma 2 to Algorithm 1 shows that it is also blocking probability optimal. 


\section{International Symposium on Network Coding (NetCod)}

Lemma 3. The blocking probability of a throughput optimal scheme is given by

$$
P_{b}^{s}=\frac{(\lambda T)^{N} / N !}{\sum_{i=0}^{N}(\lambda T)^{i} / i !} .
$$

In a system with infinite $\mathrm{I} / \mathrm{O}$ access bandwidth all drives are always available for read. Then there is no need to store more than one copy of each file chunk. That is, $\left|\mathcal{M}_{i}^{d}\right|=1$ for all $f_{i} \in \mathcal{F}$ suffices for throughput optimality.

Remark 1. Serving the earliest undecoded chunk of the leader is not essential for the optimality of the algorithm. Selecting any undecoded chunk by the leader will suffice. However, by serving undecoded chunks of the leader in a contiguous way, we promote better in-order delivery to the application.

This verifies the intuitive result that NCS does not provide benefit over UCS in an infinite I/O access bandwidth system. Note that Algorithm 1 can be adjusted to operate with coded storage via simple modifications.

\section{B. Finite I/O access bandwidth systems}

In this subsection we consider systems with drives that can become busy owing to serving other requests, i.e., drives with finite I/O access bandwidth for which $P_{b}^{D}>0$. We still assume ideal chunk transport medium with no erasures and broadcast capabilities to all active users, such as TCP for multicast variants, Ethernet, or emulated broadcasting systems.

1) Uncoded Scheme: In the finite I/O case, the concept of leaders needs modification depending on what chunks are available for access. We then distinguish between a true leader and a temporary leader in our modified scheduling algorithm. This is to handle temporary unavailability of drives that store undecoded chunks demanded by the true leader. We modify Algorithm 1 to find undecoded chunks of the true leader that are available for read. If no such undecoded chunk for the true leader is available, then we will limit our search to the next leading user and the undecoded chunks of that user, which by the approach of the service scheme must have been all decoded by the excluded leader. We continue until we can find one user who is leading among the remaining users and for whom one of its undecoded chunks is available for read. The modified scheme operates as per Algorithm 2.

2) Coded Scheme: The proposed scheme for NCS finite I/O bandwidth systems is similar to Algorithm 2 in terms of finding temporary leaders depending on drive availability. The main difference with Algorithm 2 is the choice of the chunk for service: The scheduler needs to keep track of coded chunks so far received by the users.

For each timeslot $t$, we define a binary coded chunk reception vector of size $H$ for user $u_{m}$, denoted by $\boldsymbol{q}_{m}(t)$, as follows: $q_{m, r}(t)=0$ if coded chunk $c_{r}$ has been so far received by user $u_{m}$ and $q_{m, r}(t)=1$ otherwise. Algorithm 3 describes the scheme.

3) Schemes Analysis and Comparison: In a finite I/O storage system neither UCS nor NCS can guarantee throughput optimality, since we can always find a drive unavailability
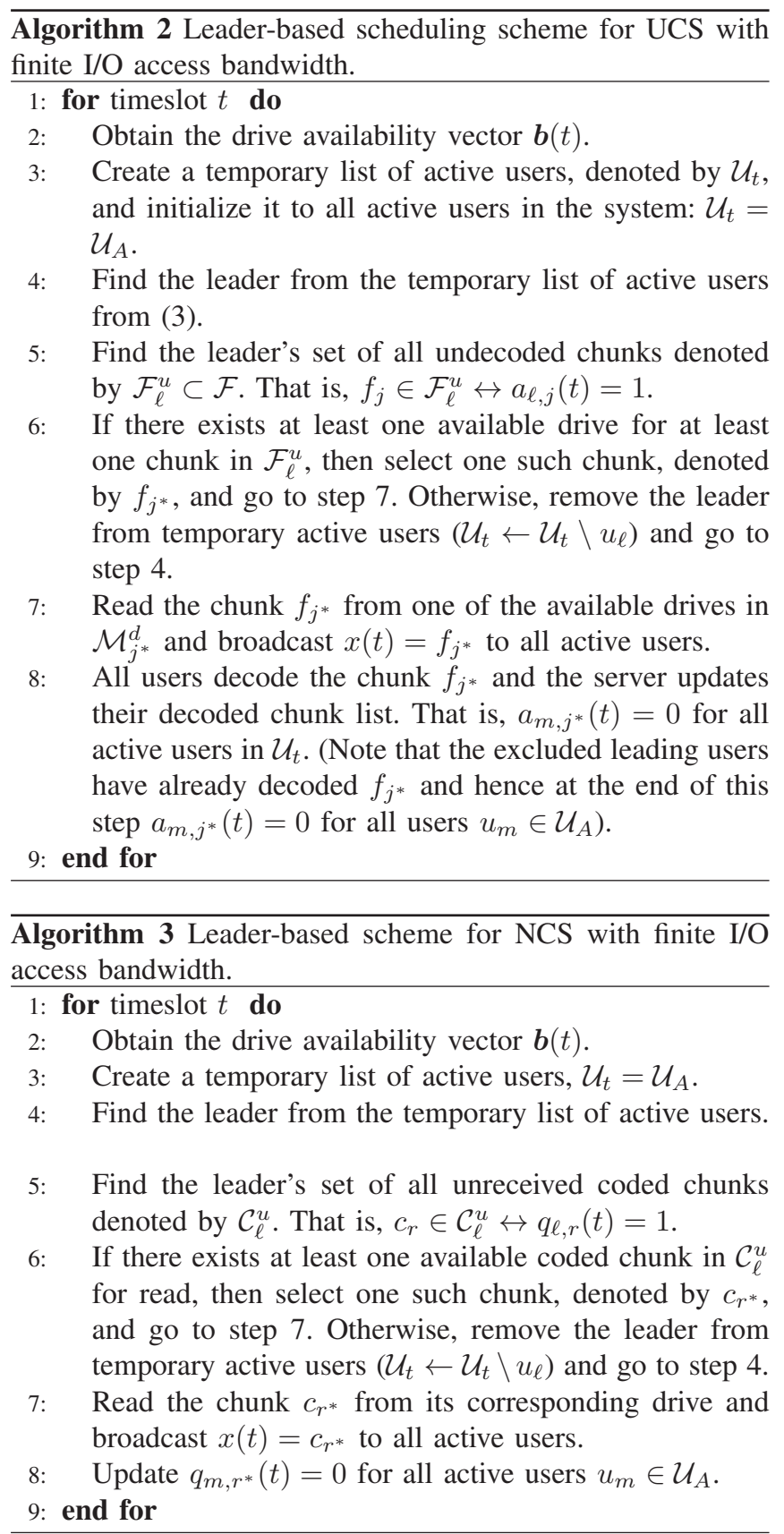

pattern with non-zero probability of occurring that would block at least one user (for instance, consider the simple case when all drives are blocked in the same timeslot). We now show simple proofs showing that while both uncoded schedule of Algorithm 2 and coded schedule of Algorithm 3 achieve maximal throughput across their respective data storage formats, that the number of targeted users using NCS with maximal throughput scheduling is at least as high as that in the UCS system.

Lemma 4. Algorithms 2 and 3 achieve maximal throughput across their respective data storage formats. 


\section{International Symposium on Network Coding (NetCod)}

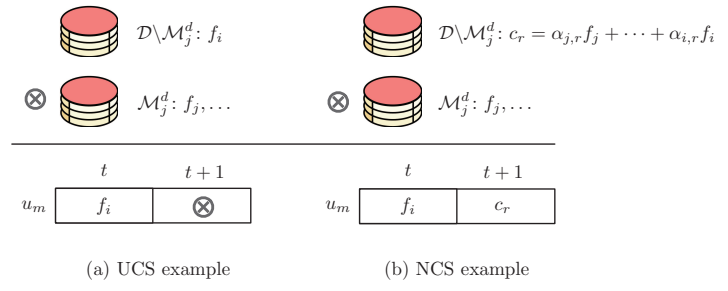

Fig. 2. Given an instance of UCS, an example construction NCS can improve throughput compared to a UCS.

Lemma 5. The number of targeted users $\left|\mathcal{U}_{n}(t)\right|$ in a finite I/O storage system using NCS with maximal throughput scheduling can be at least as high as that in a UCS system with maximal throughput scheduling.

Given any instance of UCS, we need to show (1) that no drive blocking patterns exist where the number of targeted users $\left|\mathcal{U}_{n}(t)\right|$ is higher than that in all instances of NCS, and (2) that there exist drive blocking patterns for which $\left|\mathcal{U}_{n}(t)\right|$ in NCS is higher than that in the UCS instance. The proof of (1) is in [19]. For (2), we proceed by counterexample. We can always consider a single active user $u_{m}$ with $r_{m}(t)=T-1$ under the UCS instance. See Fig. 2 when $r_{1}(2)=1$ and the only missing chunk of user $u_{1}$ is $f_{j}$. Assume that all drives in $\mathcal{M}_{j}^{d}$ are blocked during timeslot $t+1$. For the UCS system, $u_{m}$ cannot be targeted so $\left|\mathcal{U}_{n}\left(t_{+}\right)\right|=0$. However, in the NCS instance of the system, although $\mathcal{M}_{j}^{d}$ is blocked, any unseen coded chunks with $\alpha_{j, r} \neq 0$ stored on drives in $\mathcal{D} \backslash \mathcal{M}_{j}^{d}$ can still provide a new d.o.f. to user $u_{m}$, so $\left|\mathcal{U}_{n}(t+1)\right|=1$.

To further illustrate NCS improved blocking performance, we now focus on the internal true leader's blocking probability.

We consider regular $s$-striped storage systems [1] with a total of $R=W s$ drives and $T / s$ file chunks in each stripe set which is an integer. The following lemma gives the leader blocking probability in uncoded and coded systems.

Lemma 6. Assume that at timeslot $t$, the leader in the uncoded system has completely decoded $r$ out of s stripe sets, where $r=0, \cdots, s-1$, such that its knowledge space rank satisfies $r T / s \leq r_{\ell}(t)<(r+1) T / s$. Then, its internal blocking probability is given by

$$
P_{b}^{u}=\left(P_{b}^{D}\right)^{W s-W r}
$$

Now assume that in the coded system, the leader's knowledge space rank is also $r_{\ell}(t)$. A simple upper bound for the internal blocking probability $P_{b, u b}^{c}$ is given by

$$
\left(P_{b}^{D}\right)^{W s-r} \leq P_{b, u b}^{c}=\left(P_{b}^{D}\right)^{W s-\left\lfloor\frac{r_{\ell}(t)}{T / s}\right\rfloor}<\left(P_{b}^{D}\right)^{W s-(r+1)}
$$

And a simple lower bound $P_{b, l b}^{c}$ is given by

$$
P_{b, l b}^{c}=\left(P_{b}^{D}\right)^{W s-\max \left(0, r_{\ell}(t)-W T-W s\right)}
$$

which will deviate from the best possible blocking probability of $\left(P_{b}^{D}\right)^{W s}$ only when $W=1$ and $T-s<r_{\ell}(t)<T$.
Proof. In the uncoded system, if the leader has completely decoded $r$ stripe sets up to time $t$, there only remains $W s-W r$ useful drives for service and (5) follows.

The worst case for the coded system occurs when during $r_{\ell}(t)$ previous services of the leader, $\left\lfloor\frac{r_{\ell}(t)}{T / s}\right\rfloor$ out of $W s$ available drives were completely read off and hence are unavailable for further service, in which case (6) follows. The bounds are derived by using the inequalities $r T / s \leq r_{\ell}(t)<(r+1) T / s$.

The best case for the coded system occurs where all previous $r_{\ell}(t)$ services of the leader were uniformly read across $W s$ available drives. Therefore, until the leader's rank reaches $r_{\ell}(t)=W s(T / s-1)+1=W T-W s+1$, none of the drives are completely read off and are all available for service. Hence, we get, $P_{b, u b}^{c}=\left(P_{b}^{D}\right)^{W s}$ for $r_{\ell}(t)<W T-W s+1$. After this point, the drives become sequentially unavailable and $\left(P_{b}^{D}\right)^{W s-\max \left(0, r_{\ell}(t)-W T-W s\right)}$ follows. One can easily verify the last statement of the lemma using $r_{\ell}(t)<T$, the assumption that $T / s$ is an integer and $s \leq T / 2$.

Remark 2. Lemma 6 demonstrates the importance of drive selection in Algorithms 2 and 3, when more than one drive containing undecoded file chunks of the leader is available for read. One can think about this as memory in the system: Drive service units cease being helpful if all their content has been read. When comparing different variations of Algorithms 2 and 3, we expect that those which temporally spread reads across drives to have better average throughput.

\section{NumERICAL RESUltS}

Using typical values found in various modern systems, we present Monte Carlo simulation results comparing the performance of the proposed leader-based scheduling scheme for UCS and NCS systems. By using (4), the analytical results for the blocking probability of the proposed leader-based scheduling scheme for uncoded/coded storage with infinite I/O access bandwidth are presented. For all simulations, we use a regular striped mapping of file chunks onto drives.

Fig. 3 illustrates the external blocking probability of the proposed scheduling scheme for both uncoded and coded storage under drives' infinite and finite I/O access bandwidth conditions versus server buffer size, $N$. We see that when drives have finite I/O access bandwidth, NCS reduces system blocking probability over UCS and that the gap tends to grow with increasing buffer size.

Fig. 4 shows the average throughput and external blocking probability of the proposed schemes for various drive internal blocking probabilities, $P_{b}^{D}$. As shown, throughput and external blocking probability are improved in NCS compared with UCS as drives become more overwhelmed. In addition, we see that a small 3\% improvement in throughput renders a comparatively large improvement of $150 \%$ in external blocking probability.

The internal blocking probability of the true leader versus its knowledge space rank in the uncoded and coded storage for various drive internal blocking probabilities, $P_{b}^{D}$, is presented in Fig. 5. Here, the internal blocking probability of the true leader is much lower in the coded system compared 


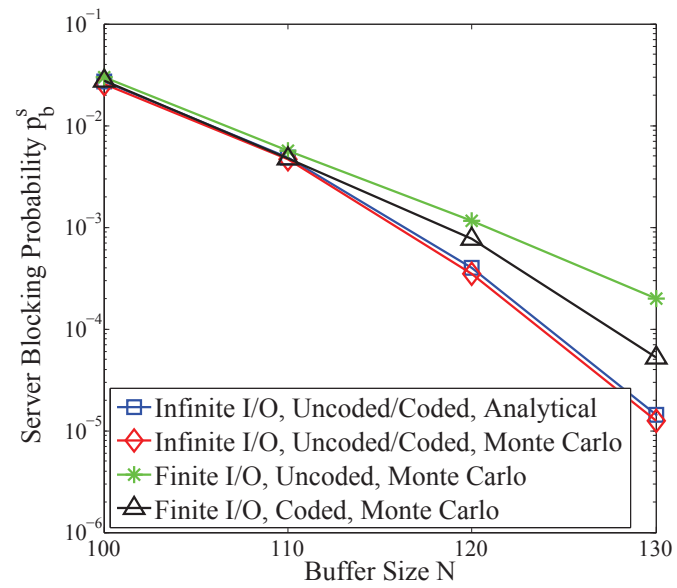

Fig. 3. Blocking probability versus server buffer size $N$ for $\lambda=0.9, T=$ $100, W=2, R=8, s=4, P_{b}^{D}=0.5$.

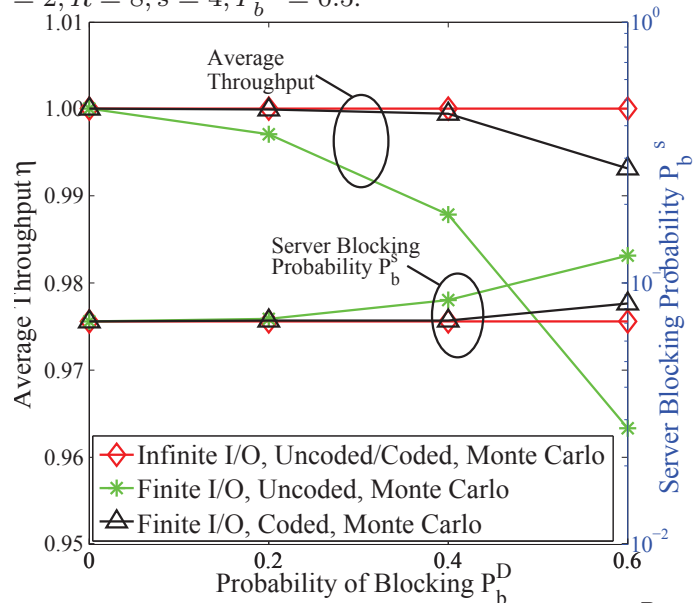

Fig. 4. Average throughput and blocking probability versus $P_{b}^{D}$ for $\lambda=$ $0.9, T=8, W=2, R=8, s=4$.

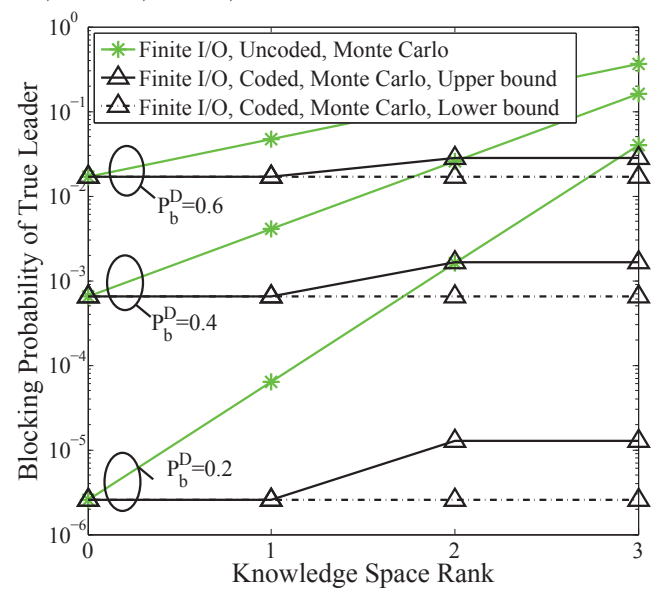

Fig. 5. Blocking probability of the true leader versus drive blocking probability $P_{b}^{D}$ for $T=8, W=2, s=4$.

with the uncoded system as the leader's knowledge space rank increases. This lower blocking probability is one factor explaining lower external blocking probability of the coded system compared to the uncoded system.

\section{Conclusions}

In this paper, we introduced a novel and simple storage model for point-to-multipoint SANs and investigated the impact of scheduling and content storage format on system blocking probability and throughput in PMP networks. We proposed two intuitive drive access scheduling techniques for both UCS and NCS systems, under infinite and finite I/O access bandwidth conditions. In finite $\mathrm{I} / \mathrm{O}$ access networks, we showed that NCS scheduling flexibility improves blocking probability and throughput over UCS. Our numerical evaluations and simulation results verify these advantages and can be used to guide future storage system design.

\section{REFERENCES}

[1] U. J. Ferner, M. Medard, and E. Soljanin, "Toward sustainable networking: Storage area networks with network coding," in Proc. Allerton Conf. on Commun., Control and Computing, Champaign, IL, Oct. 2012.

[2] A. G. Dimakis, K. Ramchandran, Y. Wu, and C. Suh, "A survey on network codes for distributed storage," Proc. IEEE, vol. 99, no. 3, pp. 476-489, Mar. 2011.

[3] D. Ford, F. Labelle, F. I. Popovici, M. Stokely, V.-A. Truong, L. Barroso, C. Grimes, and S. Quinlan, "Availability in globally distributed storage systems," in Proceedings of the 9th USENIX conference on Operating systems design and implementation, ser. OSDI'10. Berkeley, CA: USENIX Association, 2010, pp. 1-7. [Online]. Available: http://dl.acm.org/citation.cfm?id=1924943.1924948

[4] N. B. Shah, K. Lee, and K. Ramchandran, "The MDS Queue: Analysing latency performance of codes and redundant requests," CoRR, http://arxiv.org/abs/1211.5405, 2012.

[5] L. Huang, S. Pawar, Z. Hao, and K. Ramchandran, "Codes can reduce queueing delay in data centers," in Proc. IEEE Int. Symp. on Inf. Theory, Jul. 2012, pp. 2766-2770.

[6] T. E. Anderson, S. S. Owicki, J. B. Saxe, and C. P. Thacker, "High-speed switch scheduling for local-area networks," ACM Trans. Comput. Syst., vol. 11, no. 4, pp. 319-352, Nov. 1993. [Online]. Available: http://doi.acm.org/10.1145/161541.161736

[7] M. A. Marsan, A. Bianco, P. Giaccone, E. Leonardi, and F. Neri, "Multicast traffic in input-queued switches: optimal scheduling and maximum throughput," IEEE/ACM Trans. Netw., vol. 11, no. 3, pp. 465-477, Jun. 2003. [Online]. Available: http://dx.doi.org/10.1109/TNET.2003.813048

[8] B. Prabhakar, N. McKeown, and R. Ahuja, "Multicast scheduling for input-queued switches," vol. 15, no. 5, pp. 855-866, Jun. 1997.

[9] H. Yu, S. Ruepp, and M. S. Berger, "Multi-level round-robin mulitcast scheduling with look-ahead mechanism," in Proc. IEEE Int. Conf. on Commun., Kyoto, Japan, Jun. 2011, pp. 1-5.

[10] C. Feng and B. Li, Network coding: Fundamentals and applicaions, 1st ed. Academic Press, 2012, ch. Network coding for conten distribution and multimedia streaming in peer-to-peer networks.

[11] G. N. Rouskas and V. Sivaraman, "Packet scheduling in broadcast WDM networks with arbitrary transceiver tuning latencies," IEEE/ACM Trans. Netw., vol. 5, no. 3, pp. 359-370, Jun. 1997.

[12] D. Aksoy, M. J. Franklin, and S. Zdonik, "Data staging for on-demand broadcast," in Proc. 27th VLDB Conf., Roma, Italy 2001.

[13] A. Hu, "Video-on-demand broadcasting protocols: A comprehensive study," in Proc. IEEE Conf. on Computer Commun., Anchorage, AK, Apr. 2001, pp. 508-517.

[14] T. Ho, R. Koetter, M. Médard, M. Effros, J. Shi, and D. Karger, "A random linear network coding approach to multicast," IEEE Trans. Inf. Theory, vol. 52, no. 10, pp. 4413-4430, Oct. 2006.

[15] U. J. Ferner, Q. Long, M. Pedroso, L. Voloch, and M. Médard, "Building a network coded storage testbed for data center energy reduction," in Proc. IEEE SustainIT, Polermo, Italy, Oct. 2013.

[16] C.-S. Chang, W.-J. Chen, and H.-Y. Huang, "On service guarantees for input-buffered crossbar switches: a capacity decomposition approach by Birkhoff and von Neumann," in Proc. IWQoS, 1999, pp. 79-86.

[17] J. Sundararajan, S. Deb, and M. Médard, "Extending the Birkhoff-von Neumann switching strategy for multicast - on the use of optical splitting in switches," IEEE J. Sel. Areas Commun., vol. 25, pp. 36-50, 2007.

[18] M. Kim, J. K. Sundararajan, M. Médard, A. Eryilmaz, and R. Kotter, "Network coding in a multicast switch," IEEE Trans. Inf. Theory, vol. 57, no. 1, pp. 436-460, 2011.

[19] U. J. Ferner, P. Sadeghi, N. Aboutorab, and M. Médard, "Scheduling Advantages of Network Coded Storage in Point-to-Multipoint Networks," [Online]. Available: http://arxiv.org/abs/1402.3074. 